Acta Crystallographica Section D

Biological

Crystallography

ISSN 0907-4449

\section{Rouslan Moukhametzianov, ${ }^{a} \ddagger$ Manfred Burghammer, ${ }^{\mathbf{b}} \neq$ Patricia C. Edwards, ${ }^{\text {a }}$ Sebastien Petitdemange, ${ }^{b}$ Dimitri Popov, Maikel Fransen, ${ }^{a}$ Gregory McMullan, ${ }^{a}$ Gebhard F. X. Schertler $^{\mathrm{a} *}$ and Christian Riekel $^{\text {b* }}$}

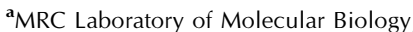
Hills Road, Cambridge CB2 0QH, England, and buropean Synchrotron Radiation Facility, BP 220, F-38043 Grenoble CEDEX, France

\# These authors contributed equally.

Correspondence e-mail:

gfx@mrc-Imb.cam.ac.uk, riekel@esrf.fr

\title{
Protein crystallography with a micrometre-sized synchrotron-radiation beam
}

For the first time, protein microcrystallography has been performed with a focused synchrotron-radiation beam of $1 \mu \mathrm{m}$ using a goniometer with a sub-micrometre sphere of confusion. The crystal structure of xylanase II has been determined with a flux density of about $3 \times 10^{10}$ photons s $^{-1} \mu \mathrm{m}^{-2}$ at the sample. Two sets of diffraction images collected from different sized crystals were shown to comprise data of good quality, which allowed a $1.5 \AA$ resolution xylanase II structure to be obtained. The main conclusion of this experiment is that a high-resolution diffraction pattern can be obtained from $20 \mu^{3}$ crystal volume, corresponding to about $2 \times 10^{8}$ unit cells. Despite the high irradiation dose in this case, it was possible to obtain an excellent high-resolution map and it could be concluded from the individual atomic $B$-factor patterns that there was no evidence of significant radiation damage. The photoelectron escape from a narrow diffraction channel is a possible reason for reduced radiation damage as indicated by Monte Carlo simulations. These results open many new opportunities in scanning protein microcrystallography and make random data collection from microcrystals a real possibility, therefore enabling structures to be solved from much smaller crystals than previously anticipated as long as the crystallites are well ordered.

\section{Introduction}

Protein microdiffraction using monochromatic beams has become a routine tool at third-generation synchrotronradiation sources (Cusack et al., 1998; Perrakis, Cipriani et al., 1999; Riekel, 2004; Riekel et al., 2005). Several protein structures have been solved using a 10 or $30 \mu \mathrm{m}$ beam on much larger crystals and improved diffraction was obtained this way, most likely owing to a lack of long-range order in the large crystals (Dutzler et al., 2002; Xiao et al., 2003). A polyhedron-like protein structure was solved from $125 \mu^{3}$ crystals by merging data from several crystals (Coulibaly et al., 2007). Beam sizes have reached the $5 \mu \mathrm{m}$ scale using a combination of focusing and collimation optics, with illuminated crystal volumes per single exposure of less than $400 \mu \mathrm{m}^{3}$, as shown for the case of bovine rhodopsin, which was refined to a resolution of $2.6 \AA$ (Li et al., 2004). More recently, the structure of a thermally stabilized recombinant rhodopsin was solved using a $5 \mu \mathrm{m}$ thick needle crystal using a diffraction volume of $125 \mu^{3}$ per pattern (Standfuss et al., 2007). The same beam size allowed the solution of an amyloid fibre structure (eight amino acids) using a diffraction volume of about $40 \mu^{3}$ (Riekel, 2004; Nelson et al., 2005).
Received 27 June 2007

Accepted 12 November 2007

PDB Reference: xylanase II, 2jic, r2jicsf.
(C) 2008 International Union of Crystallography Printed in Singapore - all rights reserved 
It has recently been suggested that a reduction of the dimension of a crystal in the horizontal scattering plane to a few micrometres should result in a reduction in secondary radiation damage owing to the high escape probability of the photoelectrons (Nave \& Hill, 2005). The use of small crystals is an interesting and desirable avenue to explore in order to extend the lower limit of accessible crystal volumes, but has several consequences for beamline instrumentation. The beam size has to be reduced by focusing to the micrometre scale in order to increase the flux density. The sphere of confusion of the rotation axis at the sample position should be less than the beam size in order to keep the sample in the beam during data collection. Owing to the limiting radiation dose of crystals (Henderson, 1990), one also has to be able to distribute the overall dose by multiple exposures across a crystal or a number of small crystals by a combination of mapping and rotation (Riekel et al., 2005; Nave \& Hill, 2005). In the extreme case, a random orientational distribution of crystal-

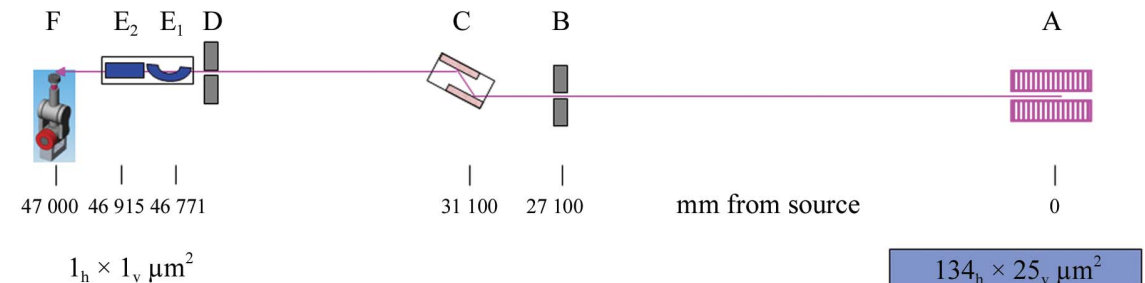

$$
1_{\mathrm{h}} \times 0.35_{\mathrm{v}} \mathrm{mrad}^{2}
$$$$
0.2_{\mathrm{h}} \times 0.02_{\mathrm{v}} \mathrm{mrad}^{2} \rightleftharpoons
$$

\section{Figure 1}

Schematic design and distance indications of the undulator source and optics. A, low- $\beta$ undulator source; $\mathrm{B}$, primary slits; $\mathrm{C}$, double $\mathrm{Si}(111)$ monochromator; $\mathrm{D}$, secondary slits in front of a Kirkpatrick-Baez (KB) mirror; $\mathrm{E}_{1}$ and $\mathrm{E}_{2}, \mathrm{~KB}$ mirrors; F: micromanipulator and goniometer. The size and divergence of the beam at the undulator source point and at the focal spot are symbolized by squares. The distance from the source and the beam divergence are indicated.

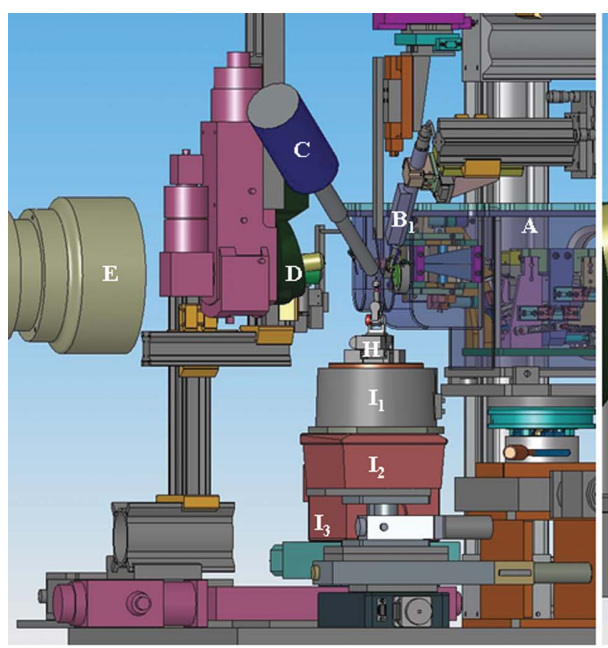

(a)

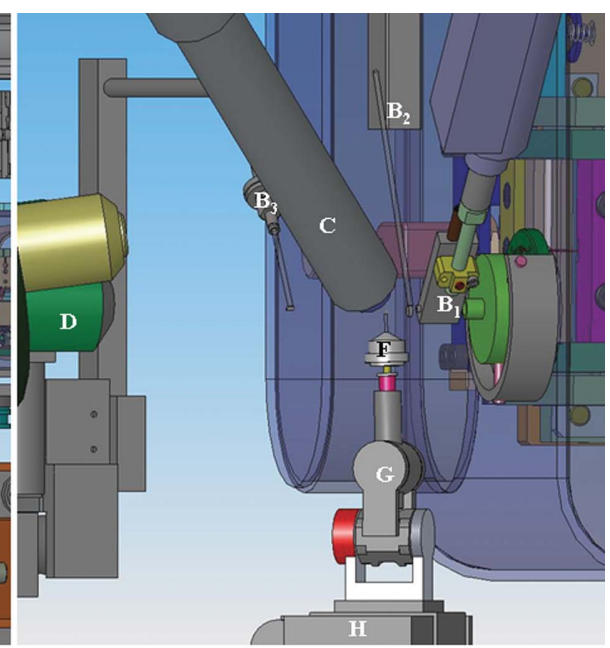

(b)

\section{Figure 2}

Schematic design of the goniometer setup during sample alignment. (a) Overall view; $(b)$ close-up of the sample environment. Components: $A$, mirror chamber; $\mathrm{B}_{1}$, ionization chamber; $\mathrm{B}_{2}$, guard aperture support; $\mathrm{B}_{3}$, beam stop; C, cryoflow; D, microscope objectives; E, MAR 165 CCD; F, sample support; $\mathrm{G}$, micromanipulator; $\mathrm{H}, x y$ sample stage; $\mathrm{I}_{1}-\mathrm{I}_{3}, x y z$ sample positioner. For data collection the microscope is translated vertically into a home position and the MAR CCD is translated to a chosen distance from the sample. lites could be exploited instead of an active rotation. In ition, the instrument has to be optimized for a low scatbackground in order to maximize the signal-to-noise fully optimized for backround possibilities for scanning a sample through the beam (Riekel $e t$ , 2005) micro-collimating system developed for scanning AXS/WAXS experiments (Fig. 1; Riekel, 2000). Here, pattern. A piezo-micromanipulator combined with an airbearing rotation axis provided the precision required to centre the crystal in the micrometre-sized X-ray beam (Fig. 2).

In the current study, xylanase II crystals were selected to assess the applicability of a micrometre-sized beam to protein crystallography (Fig. 3). Xylanase depolymerizes xylan molecules into monomeric units that are used by bacteria and fungi, and xylanase producers are typically found in environments in which plant material is deposited (Torronen, Harkki et al., 1994; Torronen, Rouvinen et al., 1994). Recently, there has been interest in xylanases for the development of environmentally friendly technologies in the paper and pulp industry (Kulkarni et al., 1999; Polizeli et al., 2005). Bleaching paper pulps with xylanases is a commercial application for these enzymes (Clarke et al., 2000).

Xylanase II from the fungus Trichoderma reesei is readily available and can be crystallized rapidly, producing very well diffracting crystals. In this study, we show that a well defined high-resolution structure from xylanase II can be obtained with the $1 \mu \mathrm{m}$ setup at the ESRF and we discuss how the beam geometry could be responsible for a possible reduction in radiation damage.

\section{Methods}

\subsection{Microfocus setup}

Experiments were performed at the ESRF ID13 beamline using an invacuum low- $\beta$ undulator optimized for X-ray energies of about $13 \mathrm{keV}$ (Fig. 1). The beam is shaped by water-cooled 
primary slits, monochromated to $\lambda=0.976 \AA$ by a liquid- $\mathrm{N}_{2}$ cooled $\mathrm{Si}(111)$ double crystal and focused by crossed Ptcovered Si mirrors. While practically the full vertical beam was accepted, the horizontal beam size was reduced by about a factor of three using slits at the entrance of the KirkpatrickBaez (KB) mirror system in order to obtain an approximate matching of horizontal and vertical size and divergence at the focal spot. The beam intensity was monitored with a microionization chamber in front of the sample. The exit of the ionization chamber is fitted with a $20 \mu \mathrm{m}$ guard aperture, which removes stray radiation generated by the KB mirror. An additional $200 \mu \mathrm{m}$ lead aperture is placed between the crystal and the exit of the ionization chamber to further improve the background on the detector. A combination of micro-apertures is also used for micro-small-angle scattering experiments (Riekel, 2000). An effective beam size of about $1 \times 1 \mu \mathrm{m}$ (measured at half-maximum) was determined by a knife-edge scan of the guard aperture at the focal spot position with a beam divergence of about $1_{\text {hor }} \times 0.35_{\text {vert }}$ mrad (Fig. 1 ).

Focusing by highly demagnifying $\mathrm{KB}$ mirrors is known to generate diffuse tails. However, we found no evidence for additional scattering in the diffraction patterns. However, the possible generation of consequences arising from such diffuse tails will have to be studied in the future in more detail. After the crystal, a micro-beamstop of lead is placed just outside the cryostream as close as possible to the crystal in order to reduce the background from air scattering of the primary beam. In such a setup the flux density at the sample within an $\sim 1 \times$ $1 \mu \mathrm{m}$ beam reaches up to $3 \times 10^{10}$ photons s ${ }^{-1} \mu \mathrm{m}^{-2}$.

In view of the small beam size, a microgoniometer setup with a sphere of confusion smaller than the beam size and optimum sample observation was required (Fig. 2). We used a vertically oriented MICOS UPR160 air-bearing spindle (company specifications: $100 \mathrm{~nm}$ eccentricity; about $2.5 \mu \mathrm{rad}$ wobble at the level of the rotation axis), a MICOS MT55 $x y$ microstage and a Kleindiek MM3A micromanipulator carrying a Hampton Research magnetic base. The sample was located about $110 \mathrm{~mm}$ above the rotation-axis bearings, which introduces a rotational eccentricity of about $0.3 \mu \mathrm{m}$ at the sample position. The microgoniometer setup was positioned by a large-stroke $x y z$ stage. An Olympus microscope with motorized rotating turret looking upstream along the beam path on the focus position was used for sample alignment in the focal spot. The microscope was translated out of the beam for data collection by a vertical motorized stage. Alignment of the air-bearing spindle axis with the microbeam was achieved with a precision of about $1 \mu \mathrm{m}$ by using fibrediffraction patterns from the $20 \mu \mathrm{m}$ nylon loop. The marked beam position

Figure 3 was stable for the duration of the experiment. Beam and optical alignments of the crystal were carried out with a new software interface specifically designed for the scanning microgoniometer.

\subsection{Crystallization}

Xylanase $(21 \mathrm{kDa})$ from $T$. longibrachiatum (previously known as $T$. reesei) was purchased from Hampton Research (HR7-106). The sample was diluted to $10 \mathrm{mg} \mathrm{ml}^{-1}$ in sterilefiltered deionized water, clarified by centrifugation at $12000 \mathrm{~g}$ for $15 \mathrm{~min}$ at $277 \mathrm{~K}$ and used immediately after preparation. $100 \mathrm{nl}$ sitting-drop crystallizations were set up in an MRC UV transparent 96-well plate (Wilden; Molecular Dimensions) using an Innovadyne dispensing robot and incubated at $291 \mathrm{~K}$. Plates were monitored for crystal formation with the MRC multiwavelength imaging system at 380 and $280 \mathrm{~nm}$ (Fig. 3a). The crystal image at $280 \mathrm{~nm}$ showed strong UV absorption, but UV inspection did not affect diffraction quality. Crystals formed across a range of ammonium sulfate concentrations and varying $\mathrm{pH}$ values. The crystals used for this data collection were grown in $2 M$ ammonium sulfate, $100 \mathrm{mM} \mathrm{MES} \mathrm{pH}$ 5.5 and $0.5 \mathrm{M} \mathrm{MgCl}_{2}$.

Two different sized crystals were chosen from the same crystallization drop and frozen in liquid nitrogen with the addition of $20 \%$ glycerol in nylon loops (Hampton Research, HR8-126). The $180 \times 180 \times 30 \mu \mathrm{m}\left(9.7 \times 10^{5} \mu \mathrm{m}^{3}\right)$ size of the larger crystal is very common for X-ray protein crystallography and the $20 \times 20 \times 70 \mu \mathrm{m}\left(=2.8 \times 10^{4} \mu \mathrm{m}^{3}\right)$ size of the smaller crystal is in the upper volume range of crystals typically used for microcrystallography at present (Riekel et al., 2005).

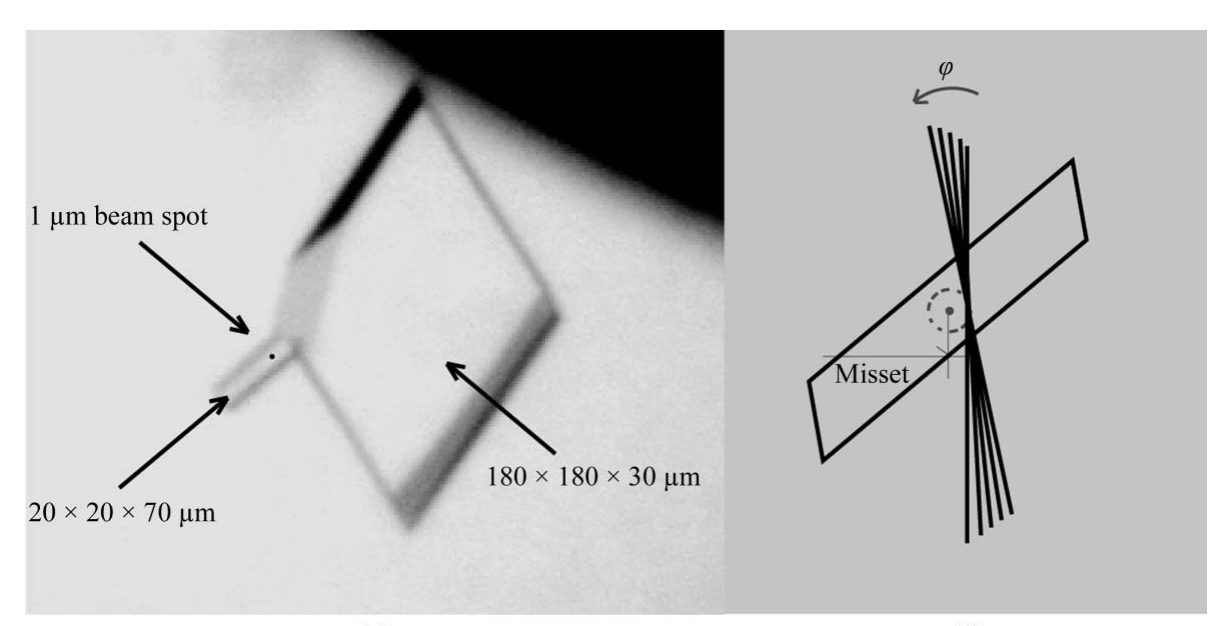

(a) (b)

Crystal/beam relationship. (a) Large and small crystals used for data collection with dimensions of $180 \times 180 \times 30$ and $20 \times 20 \times 70 \mu \mathrm{m}$, respectively; $1 \mu \mathrm{m}$ beam spot. $(b)$ Strategy for data acquisition. After every rotation, new material is present in the narrow beam channel. A small intentional misset $(2-5 \mu \mathrm{m})$ of the rotation axis was introduced. During data collection this creates a cylinder around the rotation centre, with the multiply illuminated area lying on the surface of the cylinder. Consequently, this minimizes the inclusion of damaged crystal volume contributing to individual diffraction patterns during total exposure. 


\subsection{Diffraction data collection}

Data were collected with exposure times of $1 \mathrm{~s}$ per image with $0.5^{\circ}$ oscillations using a $1 \mu \mathrm{m}$ beam with a beam divergence of $1 \mathrm{mrad}$ horizontally and $0.35 \mathrm{mrad}$ vertically (Fig. 1). Diffraction patterns were recorded on a MAR CCD 165 detector while the crystal was kept in a $100 \mathrm{~K}$ cryostream flow. The crystal-to-detector distance was determined to be $99.8 \mathrm{~mm}$ by calibration using $\mathrm{Al}_{2} \mathrm{O}_{3}$ powder. The large crystal diffracted well beyond $1.5 \AA$, but the actual resolution limit was not determined. For the small crystal, we set the limit to $1.5 \AA$, where there was a good signal-to-noise ratio for the outer reflections $[I / \sigma(I)=2.5] .180^{\circ}$ of data were collected from the large crystal. However, there was only enough time to collect $90^{\circ}$ of data from the smaller crystal.

The $1 \mu \mathrm{m}$ beam (see $\$ 2.1$ ) illuminates crystal volumes of 20 $30 \mathrm{~m}^{3}$. Accordingly, a high flux of about $3 \times 10^{10}$ photons s$^{-1}$ is needed to record a single diffraction image to high resolution. If conventional measurements were performed at such a high flux an accumulation of radiation damage would quickly occur within the illuminated volume, which in this case would be used for the recording of many images. In our case, however, each oscillation image measured with the microbeam exposes a fresh narrow channel of the crystal and therefore a high-quality pattern is obtained each time. Overlap was minimized by an intentional misset of the rotation axis $(2-5 \mu \mathrm{m})$. A cylinder around the rotation centre is created during data collection, with the multiply illuminated areas lying on the surface of the cylinder (Fig. 3b).

It is only important that the illuminated volume contributing to a single image does not suffer from too extensive a dose of X-rays. Excellent high-resolution diffraction patterns from both crystals were acquired with diffraction spots clearly visible at $1.5 \AA$ using the unattentuated beam (Fig. 4).

\section{Results}

\subsection{Data processing}

We collected complete data from the large crystal $\left(>180^{\circ}\right)$ with a completeness that was well within the recommended range at $99.9 \%$; however, some reflections in the lowresolution range were overloaded and rejected. We decided to collect a large wedge of data from the much smaller crystal (Fig. 3a) in order to compare data statistics. Because of time pressure, we were only able to collect $90^{\circ}$ of data from the smaller crystal, but we did not intend to solve the structure from this data. The completeness for the small crystal was $86 \%$ for the inner and outer shell, which indicates that a full data set would have had excellent completeness. Finally, we combined the two data sets and obtained an exceptionally good completeness for all resolution shells.

Data were processed with MOSFLM and SCALA (Collaborative Computational Project, Number 4, 1994). DPS autoindexing (Steller et al., 1997) in MOSFLM (Leslie, 1992) using data from single images as well as from several images clearly indicated a monoclinic Laue group for the crystals, with unit-cell parameters $a=40.57, b=38.96, c=57.46 \AA, \beta=110.75^{\circ}$ for the smaller crystal and $a=40.57, b=38.83, c=57.34 \AA$, $\beta=110.61^{\circ}$ for the larger crystal after cell-parameter refinement using two segments of data separated by $90^{\circ}$. The mosaic spread for both crystals was estimated to be $0.3^{\circ}$. Data were successfully integrated in MOSFLM while refining crystal, beam and detector parameters using the least-squares procedure for each image. Refined beam and detector parameters

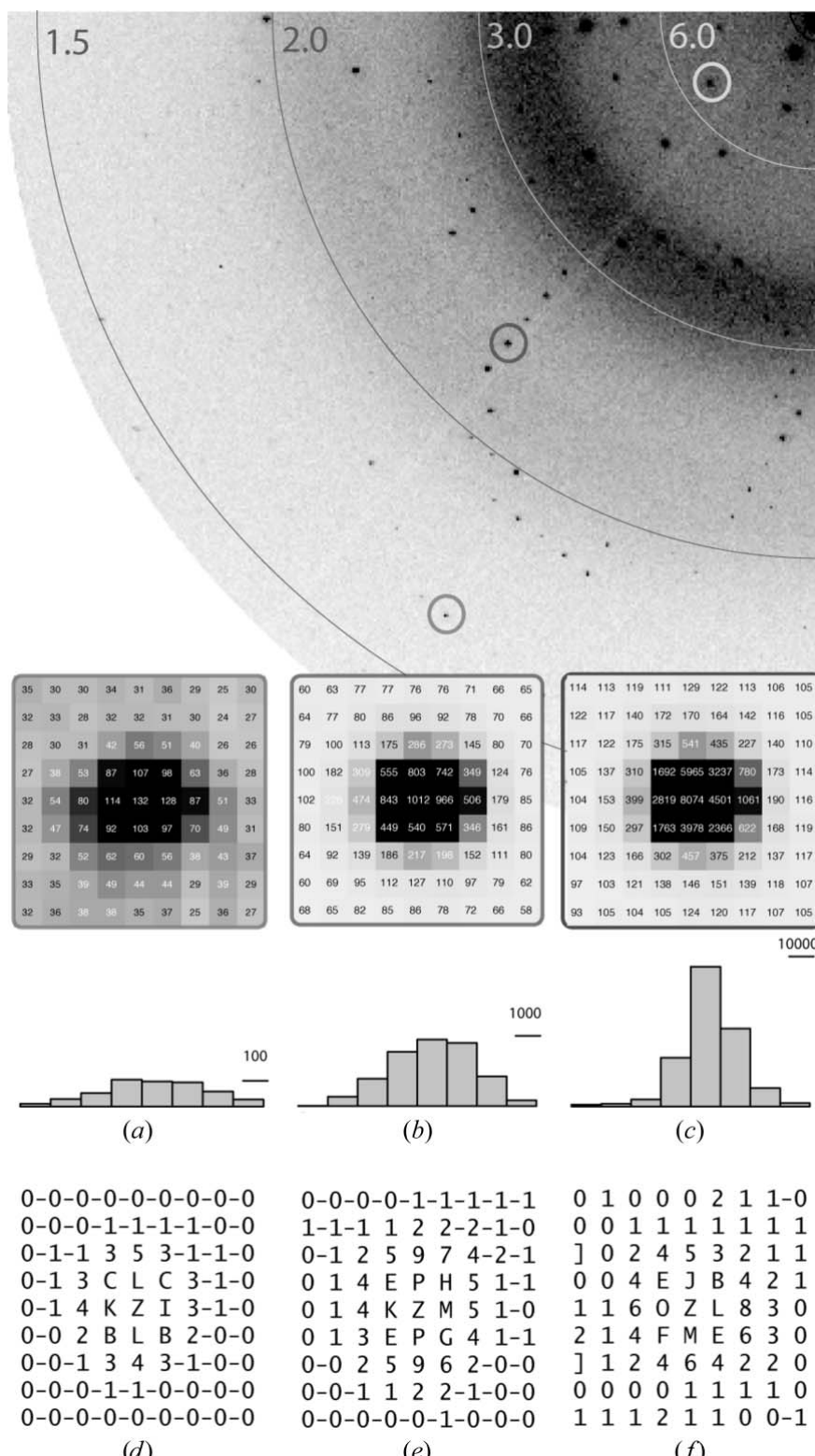

(d)

(e)

(f)

Figure 4

Diffraction pattern of xylanase II. $(a),(b),(c)$ : Spot shapes for reflections of high, medium and low resolution, respectively, with different magnitude of counts. $(d),(e),(f)$ : Normalized standard profiles (background pixels, 0 ; peak pixels are shown as a histogram within the range $\{1-9$, A-Z $\}$ ) accumulated in MOSFLM from spots of several adjacent images within a certain detector area. The detector pixel size is $78.94 \times$ $78.94 \mu \mathrm{m}$. The shape of low- and medium-resolution spots is mainly defined by the detector point-spread function. Average profiles do not adequately fit the individual spot shape for strong spots at low resolution, therefore in this resolution range integration by summation of pixels is superior to profile fitting. A weighted integration scheme described in the text was used to find the best compromise. 
showed no significant variation within the whole data collection, demonstrating the stability of the position of the micrometre-sized beam at the detector. A slight gradual sliding of the crystal indicated by a change in crystal missetting angles during orientation refinement reached about $0.7^{\circ}$ for a total $\varphi$ range of $180^{\circ}$. This could well arise from small gradual shifts of the crystal mounting loop during crystal rotation owing to the magnetic junction to the goniometer or could be a consequence of a very small deviation of the angle between the spindle and beam from $90^{\circ}$, but is irrelevant for data processing.

The extremely small beam size allows very sharp diffraction spots to be recorded; under experimental conditions, the spot shape on the image is mainly defined by the detector pointspread function. The actual spot size for low- and mediumresolution reflections, as estimated from the value of the mosaic spread, is well within the pixel size of the detector $(79 \mu \mathrm{m})$ and therefore the profile of these spots is very similar over a wide range of resolution (Fig. 4). Only the profile of weaker high-resolution spots differs through the diffraction image and contains information about the mosaicity of the crystal and the degree of reflection partiality, which could be useful if the detector point-spread function was low and the pixel size was small. The average spot profiles accumulated from spots of several successive images in MOSFLM are very much the same for different detector regions. Taking these observations into account, we used a weighted integration scheme to optimally integrate the reflections. For strong reflections with insignificant random noise the use of average spot profiles for integration of reflection intensities $\left(I_{\text {prof }}\right)$ becomes slightly worse than summation integration $\left(I_{\text {sum }}\right)$, as indicated by $R_{\text {merge, }}$ which follows from the fact that the peak area spreads over only very few detector pixels (Figs. $4 c$ and $4 f$ ). For weak reflections, the use of profile fitting is preferred since it is significantly better than summation integration with respect to the reduction of random noise (Figs. $4 a$ and $4 d$ ). Finally, the intensity of reflection was set to the weighted sum of the profile-fitted intensity and summation-integration intensity in SCALA as follows

$$
I=w I_{\text {prof }}+(1-w) I_{\text {sum }},
$$

where the weight $w=1 /\left[1+\left(I_{\text {sum }} / I_{\text {mid }}\right)^{3}\right]$ and $I_{\text {mid }}$ is the average intensity of all reflections.

Comparison of scaling and merging in SCALA for two crystals shows that in the case of the larger crystal there is a substantial difference in diffraction strength between 'face-on' and 'edge-on' orientations of the crystal (up to a factor of ten); this is primarily a consequence of the plate-like crystal shape, which causes a large change in the X-ray channel volume irradiated by the microbeam. The same difference in diffraction strength would occur for a conventional beam roughly matching the crystal size. However, for the conventional beam the reason is the change in the cross-section of the X-ray beam contributing to the diffraction. Merging of scaled strong signal-to-noise reflections in the low-resolution range shows less agreement between symmetry-related reflections for the
Table 1

Data-collection and refinement statistics.

Values in parentheses are for the highest resolution shell.

\begin{tabular}{|c|c|c|c|}
\hline & Large crystal & Small crystal & Merged data \\
\hline \multicolumn{4}{|l|}{ Data collection } \\
\hline Space group & $P 2_{1}$ & $P 2_{1}$ & $P 2_{1}$ \\
\hline \multicolumn{4}{|c|}{ Unit-cell parameters } \\
\hline$a(\AA)$ & 40.57 & 40.57 & 40.57 \\
\hline$b(\AA)$ & 38.83 & 38.96 & 38.89 \\
\hline$c(\AA)$ & 57.34 & 57.46 & 57.40 \\
\hline$\alpha\left(^{\circ}\right)$ & 90.0 & 90.0 & 90.0 \\
\hline$\beta\left(^{\circ}\right)$ & 110.61 & 110.75 & 110.75 \\
\hline$\gamma\left({ }^{\circ}\right)$ & 90.0 & 90.0 & 90.0 \\
\hline Resolution $(\AA)$ & $1.50(1.50-1.54)$ & $1.50(1.50-1.54)$ & $1.50(1.50-1.54)$ \\
\hline$R_{\text {merge }}$ & $0.066(0.162)$ & $0.064(0.354)$ & $0.084(0.265)$ \\
\hline$I / \sigma(I)$ & $14.3(7.1)$ & $10.1(2.5)$ & $16.8(6.8)$ \\
\hline Completeness (\%) & $99.7(99.9)$ & $86.8(86.8)$ & $99.9(100.0)$ \\
\hline Redundancy & $3.6(3.4)$ & $2.2(2.0)$ & $5.5(5.3)$ \\
\hline \multicolumn{4}{|l|}{ Refinement } \\
\hline Resolution $(\AA)$ & 1.5 & 1.5 & 1.5 \\
\hline No. of reflections & 26748 & 22338 & 26925 \\
\hline \multirow{2}{*}{\multicolumn{4}{|c|}{$\begin{array}{l}\Lambda_{\text {work }} / I_{\text {free }} \\
\text { No. of atoms }\end{array}$}} \\
\hline & & & \\
\hline Protein & 1472 & 1472 & 1472 \\
\hline Water & 122 & 122 & 122 \\
\hline \multicolumn{4}{|l|}{$B$ factors $\left(\AA^{2}\right)$} \\
\hline Protein & 11.4 & 11.5 & 11.5 \\
\hline Water & 21.4 & 21.4 & 21.4 \\
\hline \multicolumn{4}{|c|}{ Overall anisotropic $B\left(\AA^{2}\right)$} \\
\hline$B_{11}$ & & & 1.34 \\
\hline$B_{22}$ & & & 0.27 \\
\hline$B_{33}$ & & & -1.62 \\
\hline$B_{13}$ & & & 0.76 \\
\hline \multicolumn{4}{|l|}{ R.m.s. deviations } \\
\hline Bond lengths $(\AA)$ & 0.005 & 0.005 & 0.005 \\
\hline Bond angles $\left({ }^{\circ}\right)$ & 1.4 & 1.4 & 1.4 \\
\hline
\end{tabular}

larger crystal $\left(R_{\text {merge }}=0.057,10-4 \AA\right)$ than for the smaller one $\left(R_{\text {merge }}=0.040,10-4 \AA\right)$.

The data from both the large and the small crystals could be satisfactorily merged with improved completeness, especially at low resolution, and good multiplicity, because some overloaded reflections from the large crystal were measured correctly for the smaller crystal. A slight increase in $R_{\text {merge}}$, which could arise from more data being merged or be a consequence of non-isomorphism, was observed. The final merging statistics, data redundancy and completeness for three data sets are shown in Table 1 . The overall Wilson $B$ factor is not changed, indicating no significant radiation damage.

Statistical analysis of the intensity distribution of diffraction data was performed with TRUNCATE (CCP4 suite) and showed no deviations from expected intensity distributions.

\subsection{Refinement}

The structure of xylanase II was solved by molecular replacement. Several structures of xylanase II from crystals belonging to different space groups have been deposited in the Protein Data Bank (Moiseeva \& Allaire, 2004; Watanabe et al., 2006). The model selected for molecular replacement was a $2 \AA$ resolution X-ray structure solved from a crystal form identical to that in the present work (Miyatake et al., 2006). 
Molecular replacement was performed using MOLREP (Collaborative Computational Project, Number 4, 1994; Vagin \& Teplyakov, 1997). The structure from the PDB (entry 2d97) was reduced to a polyalanine model in order to decrease model bias and was used as a search model. Diffraction data in the resolution range 10-3 $\AA$ were used in the rotation and translation functions. A single distinct rotation-function peak was found and after a translation-function search the solution with the highest score, which was well above the others, was selected. Since xylanase II consists of 190 amino acids, there is only one molecule in the asymmetric unit, with $54 \%$ solvent content. The molecular-replacement solution for the polyalanine search model had an $R$ factor of 0.461 for the larger crystal and 0.441 for the smaller one.

Having data to $1.5 \AA$ allowed us to successfully complete the molecular-replacement polyalanine model using the $A R P /$ $w A R P$ suite in a mode alternating iterative building and refinement steps (Perrakis, Morris et al., 1999). Maximumlikelihood refinement cycles were performed in REFMAC and were combined with addition and deletion of atoms, updating the starting model in ARP (Murshudov et al., 1997). The initial polyalanine model was retraced and used only for guiding the autobuilding; therefore, model bias was under control. $5 \%$ of the data set was randomly chosen and used for crossvalidation using $R_{\text {free }}$. After only 20 refinement cycles in ARP/ $w A R P$ a fairly complete model consisting of 176 residues with side chains for the larger crystal and $R=0.190, R_{\text {free }}=0.216$ (175 residues, $R=0.195, R_{\text {free }}=0.239$ for the smaller crystal) was obtained.

The ARP/wARP model was completed using the graphics program $O$ (Jones et al., 1991). Solvent molecules with displacement parameters lower than $35 \AA^{2}$ were picked up from the difference density map. The structure was finally refined in CNS (Brünger et al., 1998). Several rounds of energy minimization were performed followed by individual temperaturefactor refinement. Refinement statistics and geometry parameters of the model are presented in Table 1 . The final model consists of 190 residues and 120 water molecules. All the residues are within allowed regions in the Ramachandran plot.

\section{Discussion}

In this study, we have used a $1 \mu \mathrm{m}$ beam setup for the first time (Figs. 1 and 2) and have determined the structure of a well diffracting crystal of xylanase II (Fig. $3 a$ ) to a resolution of $1.5 \AA$ (Table 1). We studied the diffraction from an average-sized and a small crystal. The final electron-density maps after refinement against a combined data set (Table 1) showed excellent densities for all residues, with the exception of a few that are not ordered and are located on the protein surface. In particular, those residues that are highly susceptible to radiation damage showed clear density (Fig. 5). Increasing irradiation of protein crystals results in structural damage as follows: disulfide bridges break, glutamates and aspartates decarboxylate, tyrosine residues lose their hydroxyl group and methionines lose their C-S bond (Burmeister, 2000; Weik et al., 2000). However, there was no indication of specific radiation damage from our electron-density maps for these residues, as shown in Fig. 5. Furthermore, for the smaller crystal the temperature factors for the atoms of aspartate side chains were $12.50 \AA^{2}$ on average and those for buried glutamates were $10.4 \AA^{2}$ on average, which is not substantially different from the overall average $B$ factor of $11.4 \AA^{2}$ for protein side chains. Very similar $B$ factors are observed for glutamates and aspartates in the larger crystal. No radiation damage is indicated for tyrosines and methionine. We did not intend to study radiation damage in detail or try to find the limiting dose; for this we would need to collect several data sets (Diederichs, 2006). We have demonstrated that with a high-brilliance $1 \mu \mathrm{m}$ beam we can obtain a good high-resolution structure of a medium-sized protein.

The experiments show that one can collect a diffraction pattern from an irradiated crystal volume of about $20 \mu \mathrm{m}^{3}$ using an approximately $1 \mu \mathrm{m}^{2}$ focused beam $\left(\sim 3 \times 10^{10}\right.$ photons s$\left.{ }^{-1} \mu \mathrm{m}^{-2}\right)$. As a consequence of rotation, new material will be constantly swept into the beam (Fig. 3b), allowing the collection of a complete data set without significant radiation damage (as evident from the data statistics). In spite of the high irradiation dose, it was possible to obtain an excellent high-resolution map. The irradiated volume of a

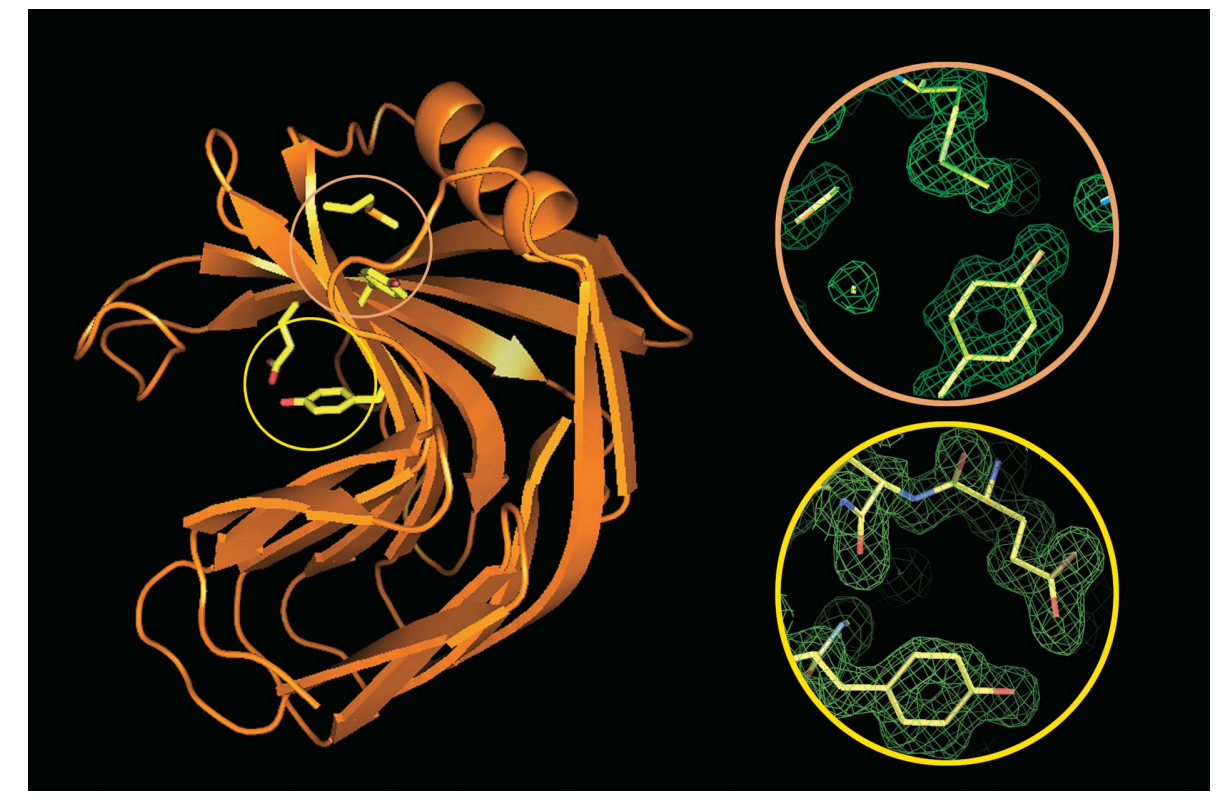

Figure 5

Xylanase II structure. Left, secondary structure with selected residues; right, selected residues with $1.5 \AA$ resolution $\sigma_{\mathrm{A}}$-weighted $2 F_{\mathrm{o}}-F_{\mathrm{c}}$ electron-density map contoured at $0.7 \mathrm{e} \AA^{-3}$ level. The excellent densities for beam-sensitive residues, for example glutamic acids, tyrosines and methionine, provide no evidence of radiation damage. 
single exposure corresponds to about $2 \times 10^{8}$ unit cells and is therefore a factor of 5-15 smaller than the previously estimated limiting number of unit cells required to record a highresolution diffraction pattern for $1^{\circ}$ of rotation (Glaeser et al., 2000; Teng \& Moffat, 2000, 2002). A comparison with selected other microdiffraction studies is shown in Fig. 6. Data from inorganic crystals have been added in order to demonstrate that crystals with smaller scattering power can be refined if radiation-damage problems can be neglected. The results on xylanase II suggest that the minimum crystal volumes and scattering power required to collect a high-resolution pattern have been overestimated in previous studies (Glaeser et al., 2000; Teng \& Moffat, 2000, 2002). Crystals of lysozyme and bacteriorhodopsin of dimensions $100 \times 100 \times 20-40 \mu \mathrm{m}$ diffracting to 1.5 and $2.1 \AA$ with overall $B$ factors comparable to that of xylanase and significantly higher were studied by Teng and coworkers and Glaeser and coworkers, respectively, at conventional synchrotron beamlines (Glaeser et al., 2000; Teng \& Moffat, 2000, 2002).

The diameter of the beam used in the present study is sufficiently small that in calculating expected radiation damage the escape of photoelectrons from the scattering volume has to be taken into account (Nave \& Hill, 2005). In the absence of radiation damage, the diffraction signal is expected to be proportional to the total number of incident photons and hence the number of photoelectrons generated in

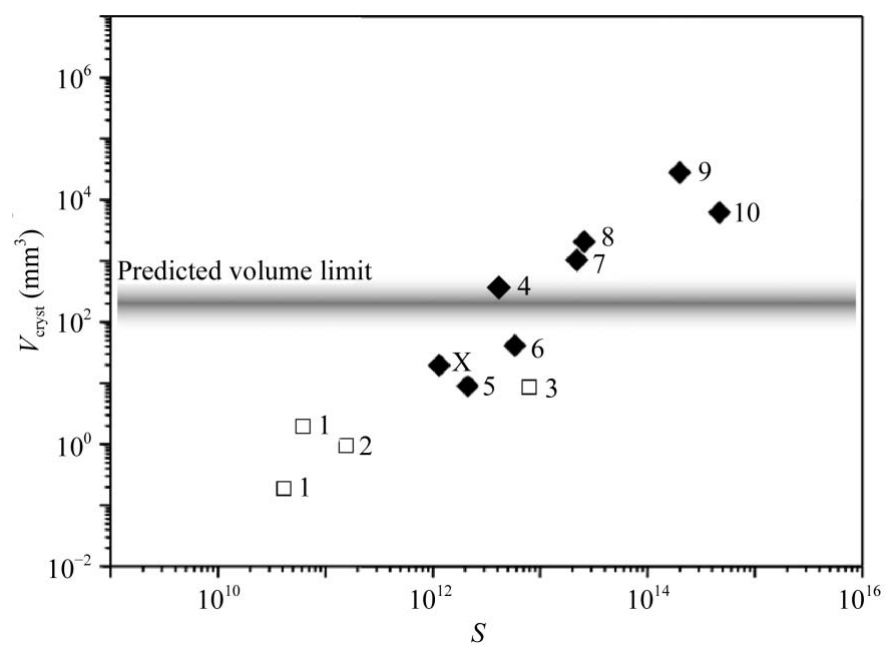

Figure 6

Irradiated crystal volume ( $\left.V_{\text {cryst }}\right)$ scaled against scattering power $(S)$ for a selected high-resolution single-crystal microdiffraction experiment (adapted from Riekel et al., 2005). The scattering power has been scaled to an average electron density: $S=\left(F_{000} / V_{\text {cell }}\right)^{2} \times \lambda^{3} \times V_{\text {cryst }}^{32}$, where $F_{000}$ is the zero-order structure factor, $V_{\text {cell }}$ is the unit-cell volume, $\lambda$ is the wavelength and $V_{\text {cryst }}$ is the crystal volume. For experiments with a beam size smaller than the crystal size, $V_{\text {cryst }}$ corresponds to the irradiated volume during a single exposure. The shaded zone indicates the previously predicted crystal-volume limit for protein crystallography. Open squares represent inorganic structures: $1, \mathrm{CaF}_{2}$ (Burghammer, 1997); 2, kaolimite (Neder et al., 1996); 3, birnessite (Gaillot et al., 2003). Filled diamonds represent protein crystals: X, xylanase II; 4, rhodopsin (Li et al., 2004); 5, $\alpha$-amylose (Popov et al., 2006); 6, amyloid-like fibrils (Nelson et al., 2005); 7, sensory rhodopsin II (Luecke et al., 2001); 8, bacteriorhodopsin (Pebay-Peyroula et al., 1997); 9, HIV-1 capsid protein (p24; Berthet-Colominas et al., 1999); 10: integrin $\alpha_{2} \beta_{1}$-binding collagen peptide (Emsley et al., 2004). a given volume. Any reduction in radiation damage with decreasing beam diameter should be reflected by an increasing proportion of photoelectron energy being deposited outside the illuminated volume. To investigate, this a standard Monte Carlo program similar to that described by Joy (1995) was used to generate trajectories and associated energy-loss distributions. In the program, directional changes are given by elastic Rutherford scattering and energy loss is calculated using the continuous slowing-down approximation. For simplicity, averaged density, atomic charge and effective mean ionization potential were used. Photoelectrons are assumed to be generated randomly within a cylinder of length $20 \mu \mathrm{m}$. For simplicity, the initial energy was taken to be that of the incident photon, while the initial direction was generated either totally randomly or taking into account the polarization of the incident beam. The results of these calculations are summarized in Fig. 7, where a small beam-enhancement factor, given by the ratio of energy deposited in the illuminated volume for a effectively infinite-diameter beam to that of finite diameter but with the same total number of photoelectrons, is plotted as a function of beam diameter and initial energies of 8.1, 12.7 and $20 \mathrm{keV}$. For a given signal, it is therefore possible to deposit less total energy in the illuminated volume by using a smaller beam diameter or higher energy photon.

A further gain in durability is expected for even smaller beams. One can still expect some further reductions in the

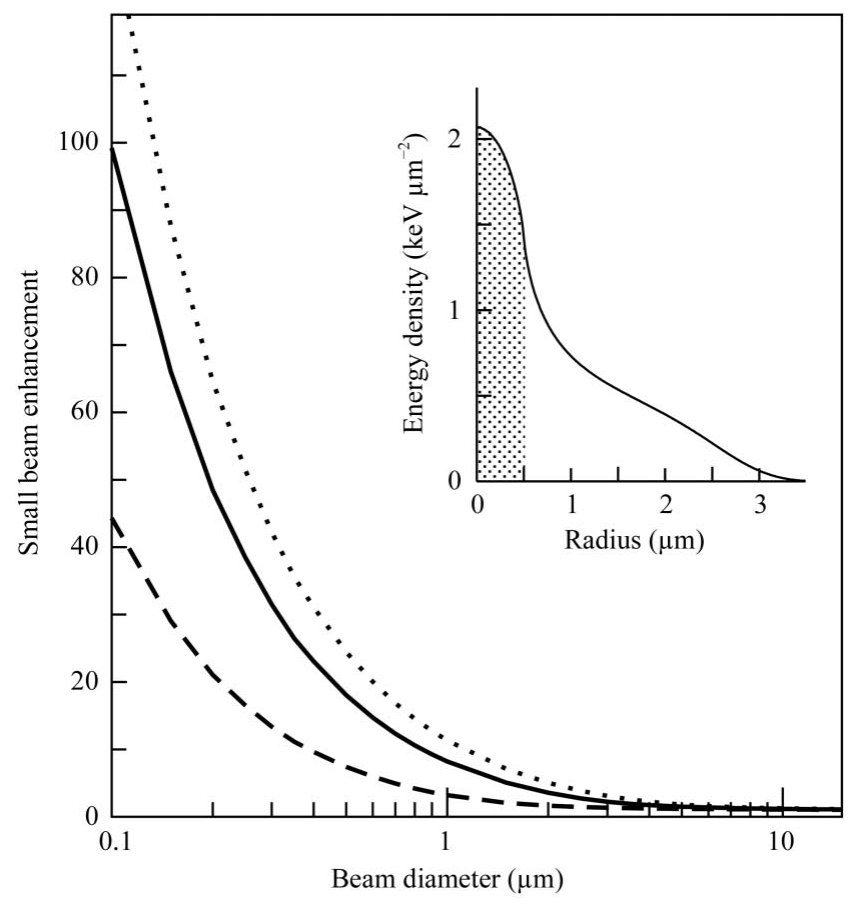

Figure 7

Calculated small beam enhancement, defined as the ratio of the total energy deposited in the crystal relative to that actually deposited in the beam volume, as a function of the beam diameter for incident X-ray energies of $8.1 \mathrm{keV}$ (dashed line), $12.7 \mathrm{keV}$ (unbroken line) and $15 \mathrm{keV}$ (dotted line). The inset shows the calculated deposited energy density per generated $12.7 \mathrm{keV}$ photoelectron as a function of radius for a $20 \mu \mathrm{m}$ thick sample illuminated by a $1 \mu \mathrm{m}$ diameter beam. On average, $12.1 \mathrm{keV}$ is deposited in the whole crystal but only $1.4 \mathrm{keV}$ of this is contained within the illuminated volume (indicated by the shading). 
scattering power necessary for data collection in experiments with micrometre- or submicrometre-sized beams applied to protein microcrystals (Fig. 6). Electron escape from the illuminated volume could explain the remarkable stability of xylanase during data collection and also the discrepancy with previously predicted smallest crystal volumes for diffraction (Fig. 6). The presence of electron escape introduces the influence of neighbouring diffracting volumes of consequent images to each other. However, the assumption of a fresh crystal volume for the next diffraction image roughly remains true because only a small fraction of electrons will escape in the next illuminated volume, with the majority of electrons escaping out of the crystal slice selected by the beam during data collection.

Our current study anticipates that protein crystals with volumes as small as $20 \mu \mathrm{m}^{3}$ could be used for structure solution. This indicates that random data collection on micrometre-size crystals presents a real opportunity. Datacollection strategies with random crystal orientation are successfully used in electron crystallography of twodimensional crystals and in the crystallography of viruses. We propose a similar strategy. Data from multiple microcrystals with random orientations could be collected using a microbeam and complete data sets merged from all of these. However, an improved microdiffraction setup and dedicated software need to be developed. For scanning crystallography to be successful, we have to assume that the crystal powder contains at least a large fraction of well diffracting but very small crystals that are isomorphous. High mosaicity will reduce the signal-to-noise ratio and lead to lower resolution. At present, we propose a scanning method that will use at least half a degree rotation per frame in order to reduce the problem with partial recorded reflections. A consequence of partial reflections is that we will need to record many more frames than in a conventional single-crystal experiment to reach an acceptable level of completeness. It is also very likely that the present indexing and scaling procedures will need to be further improved to process randomly collected data efficiently. Pixel detectors with small pixel size and reduced pointspread function will provide fast and sensitive readout of diffraction data, which will be necessary for successful scanning and random data-collection strategies. Furthermore, more detailed experimental studies on limiting factors to microcrystal diffraction making use of instrumental and X-ray optical advances are required. To this end, an advanced microgoniometer is currently under construction at the ID13 beamline nanofocus extension. With this setup, we will be able to verify if very small beams will significantly reduce the radiation-damage problem.

We gratefully acknowledge the support for RM and SP by a HFSP program grant (RG/0052). GFXS acknowledges the support of the European Commission FP6 specific targeted research project (LSH-2003-1.1.0-1). CR also acknowledges the support of the FP6 SAXIER project in acquiring the micromanipulator used in this study. We wish to thank L.
Lardiere (ESRF-ID13) for preparing Fig. 3. We wish to thank

P. Evans and A. Leslie for useful discussions.

\section{References}

Berthet-Colominas, C., Monaco, S., Novelli, A., Sibai, G., Mallet, F. \& Cusack, S. (1999). EMBO J. 18, 1124-1136.

Brünger, A. T., Adams, P. D., Clore, G. M., DeLano, W. L., Gros, P., Grosse-Kunstleve, R. W., Jiang, J.-S., Kuszewski, J., Nilges, M., Pannu, N. S., Read, R. J., Rice, L. M., Simonson, T. \& Warren, G. L. (1998). Acta Cryst. D54, 905-921.

Burghammer, M. (1997). PhD thesis, LMU.

Burmeister, W. P. (2000). Acta Cryst. D56, 328-341.

Clarke, J. H., Davidson, K., Rixon, J. E., Halstead, J. R., Fransen, M. P., Gilbert, H. J. \& Hazlewood, G. P. (2000). Appl. Microbiol. Biotechnol. 53, 661-667.

Collaborative Computational Project, Number 4 (1994). Acta Cryst. D50, 760-763.

Coulibaly, F., Chiu, E., Ikeda, K., Gutmann, S., Haebel, P. W., SchulzeBriese, C., Mori, H. \& Metcalf, P. (2007). Nature (London), 446, 97-101.

Cusack, S., Belrhali, H., Bram, A., Burghammer, M., Perrakis, A. \& Riekel, C. (1998). Nature Struct. Biol. 5, Suppl., 634-637.

Diederichs, K. (2006). Acta Cryst. D62, 96-101.

Dutzler, R., Campbell, E. B., Cadene, M., Chait, B. T. \& MacKinnon, R. (2002). Nature (London), 415, 287-294.

Emsley, J., Knight, C. G., Farndale, R. W. \& Barnes, M. J. (2004). J. Mol. Biol. 335, 1019-1028.

Gaillot, A. C., Flot, D., Drits, V. A., Burghammer, M., Manceau, A. \& Lanson, B. (2003). Chem. Mater. 15, 4666-4678.

Glaeser, R., Facciotti, M., Walian, P., Rouhani, S., Holton, J., MacDowell, A., Celestre, R., Cambie, D. \& Padmore, H. (2000). Biophys. J. 78, 3178-3185.

Henderson, R. (1990). Proc. R. Soc. London Ser. B, 241, 6-8.

Jones, T. A., Zou, J.-Y., Cowan, S. W. \& Kjeldgaard, M. (1991). Acta Cryst. A47, 110-119.

Joy, D. C. (1995). Monte Carlo Modeling for Electron Microscopy and Microanalysis. New York: Oxford University Press.

Kulkarni, N., Shendye, A. \& Rao, M. (1999). FEMS Microbiol. Rev. 23, 411-456.

Leslie, A. G. W. (1992). Jnt CCP4/ESF-EACBM Newsl. Protein Crystallogr. 26.

Li, J., Edwards, P. C., Burghammer, M., Villa, C. \& Schertler, G. F. (2004). J. Mol. Biol. 343, 1409-1438.

Luecke, H., Schobert, B., Lanyi, J. K., Spudich, E. N. \& Spudich, J. L. (2001). Science, 293, 1499-1503.

Miyatake, H., Hasegawa, T. \& Yamano, A. (2006). Acta Cryst. D62, 280-289.

Moiseeva, N. \& Allaire, M. (2004). Acta Cryst. D60, 1275-1277.

Murshudov, G. N., Vagin, A. A. \& Dodson, E. J. (1997). Acta Cryst. D53, 240-255.

Nave, C. \& Hill, M. A. (2005). J. Synchrotron Rad. 12, 299-303.

Neder, R. B., Burghammer, M., Grasl, T., Schulz, H., Bram, A., Fiedler, S. \& Riekel, C. (1996). Z. Kristallogr. 211, 763-765.

Nelson, R., Sawaya, M. R., Balbirnie, M., Madsen, A. O., Riekel, C., Grothe, R. \& Eisenberg, D. (2005). Nature (London), 435, 773-778.

Pebay-Peyroula, E., Rummel, G., Rosenbusch, J. P. \& Landau, E. M. (1997). Science, 277, 1676-1681.

Perrakis, A., Cipriani, F., Castagna, J.-C., Claustre, L., Burghammer, M., Riekel, C. \& Cusack, S. (1999). Acta Cryst. D55, 1765-1770.

Perrakis, A., Morris, R. \& Lamzin, V. S. (1999). Nature Struct. Biol. 6, 458-463.

Polizeli, M. L., Rizzatti, A. C., Monti, R., Terenzi, H. F., Jorge, J. A. \& Amorim, D. S. (2005). Appl. Microbiol. Biotechnol. 67, 577-591.

Popov, D., Burghammer, M., Buléon, A., Montesanti, N., Putaux, J. L. \& Riekel, C. (2006). Macromolecules, 39, 3704-3706.

Riekel, C. (2000). Rep. Prog. Phys. 63, 233-262.

Riekel, C. (2004). J. Synchrotron Rad. 11, 4-6. 


\section{research papers}

Riekel, C., Burghammer, M. \& Schertler, G. (2005). Curr. Opin. Struct. Biol. 15, 556-562.

Standfuss, J., Xie, G., Edwards, P. C., Burghammer, M., Oprian, D. D. \& Schertler, G. F. (2007). J. Mol. Biol. 372, 1179-1188.

Steller, I., Bolotovsky, R. \& Rossmann, M. G. (1997). J. Appl. Cryst. 30, 1036-1040.

Teng, T. \& Moffat, K. (2000). J. Synchrotron Rad. 7, 313-317.

Teng, T.-Y. \& Moffat, K. (2002). J. Synchrotron Rad. 9, 198201.

Torronen, A., Harkki, A. \& Rouvinen, J. (1994). EMBO J. 13, 2493 2501.
Torronen, A., Rouvinen, J., Ahlgren, M., Harkki, A. \& Visuri, K. (1994). J. Mol. Biol. 233, 313-316.

Vagin, A. \& Teplyakov, A. (1997). J. Appl. Cryst. 30, 1022-1025.

Watanabe, N., Akiba, T., Kanai, R. \& Harata, K. (2006). Acta Cryst. D62, 784-792.

Weik, M., Ravelli, R. B., Kryger, G., McSweeney, S., Raves, M. L., Harel, M., Gros, P., Silman, I., Kroon, J. \& Sussman, J. L. (2000). Proc. Natl Acad. Sci. USA, 97, 623-628.

Xiao, B., Spencer, J., Clements, A., Ali-Khan, N., Mittnacht, S., Broceno, C., Burghammer, M., Perrakis, A., Marmorstein, R. \& Gamblin, S. J. (2003). Proc. Natl Acad. Sci. USA, 100, 2363-2368. 